Original Research Paper

\title{
Biomimetic Finite Element Analysis Bone Modeling for Customized Hybrid Biological Prostheses Development
}

\author{
${ }^{1}$ Aversa Raffaella, ${ }^{2}$ Florian Ion T. Petrescu, ${ }^{3}$ Relly Victoria V. Petrescu and ${ }^{1}$ Apicella Antonio \\ ${ }^{1}$ Advanced Material Lab, Department of Architecture and Industrial Design, Second University of Naples, Aversa (CE) Italy \\ ${ }^{2}$ Department of Theory of Mechanisms and Robots, Bucharest Polytechnic University, Bucharest, Romania \\ ${ }^{3}$ Department of Transport, Traffic and Logistics, Bucharest Polytechnic University, Bucharest, Romania
}

\author{
Article history \\ Received: 03-10-2016 \\ Revised: 07-11-2016 \\ Accepted: 30-11-2016 \\ Corresponding Author: \\ Apicella Antonio \\ Advanced Material Lab, \\ Department of Architecture and \\ Industrial Design Second \\ University of Naples, Aversa \\ (CE) Italy \\ Email: antonio.apicella@unina2.it
}

\begin{abstract}
The human femur shows a high capacity to withstand external stresses and it is due to the mass distribution, morphology, and orthotropic behaviors of trabecular and cortical bone. Faithful modeling of the femur accounting for bone distribution and material orthotropic behavior is presented. The use of biofidel model is aimed to develop an "in silico" tool that could enable the valuation of biomechanics modification induced by the alteration of the structural and morphological characteristic in prothesized bones. Moreover, a faithful model assists us in the development of new design criteria for innovative prosthetic systems that, following the isostatic loading lines, could restore the physiological and natural stress and strains distribution. In this study a biofidel femur Finite Element Model (FEM) has been developed from Computerized Tomography (CT) scans using specific combination of software's to correctly represent bone physiology and structural behavior. Proper identification of trabecular bone arrangement and distribution in the proximal diaphysis enabled modeling and definition of material properties. The faithful femur model proposed allows us to correctly account for non-isotropic properties to the proximal end explaining the critical structural role played by trabecular bone that should be taken into account in the design of new innovative prosthetic system.
\end{abstract}

Keywords: Trabecular Bone, Biomimetic, Biomechanics, Prostheses

\section{Introduction}

The human femur has been recognized to present a specific interior structure that is characterized by a high capacity to withstand external stresses while optimizing bone mass distribution and morphology to achieve this performance (Ashman et al., 1984; Dalstyra et al., 1993). This highly efficient structural behavior is due to the orthotropic behaviors of trabecular and cortical bone (Gottesman and Hashin, 1980; Oh and Harris, 1976). The progressive physiological bone loss occurring at older age, which reduces bone toughness and capability to dissipate energy transmitted by a shock event, is the cause of aged people femur fracture. Internal structure of the femur proximal end defined by the Ward triangle is the anatomical region where the osteoporotic phenomenon alters the structural equilibrium increasing the possibility of femur neck fracture (Ashman and Rho, 1988; Burnstein et al., 1976; Carter and Hayes, 1977).
The necessity of predicting the structural modification induced by the alteration of the structural and morphological characteristic of the bone needs the development of faithful modeling of the femur accounting for bone distribution and material orthotropic behavior. This faithful model could enable us to design new prosthetic systems that restore the physiological and natural stress and strains distribution (Apicella et al., 2010: Gramanzini et al., 2016; Perillo et al., 2010, Rohlmann et al., 1982; Sorrentino et al., 2009; 2007).

A femur FEM model has been reconstructed here to correctly represent its structural behavior by modeling trabecular bone organization in the proximal end (femur head) and by defining for it material properties that are transversally isotropic. A comparison between femur model proposed and a model that allows isotropic properties to the proximal end makes evident that the first model explains structural role played by trabecular bone, defining, at the same way, a critical region in the femur neck. 


\section{Materials and Methods}

Medical Image Segmentation for Engineering application have been derived using the Mimics software (Materialise, Belgium) for processing patient medical image coming from CT. As reported in Fig. 1, processing of $\mathrm{CT}$ resulted in a highly accurate $3 \mathrm{D}$ model of the patient pelvis anatomy.

This patient-specific model has been processed to develop new prosthetic engineering applications through a combined use of Mimics and 3-Matic (Materialise, Belgium) software's.

Namely, 3D solid and Finite Element Models (FEM) have been developed to simulate the external and internal morphology of the femur and other complex bone structures accounting for the orientation and densities of the head trabecular systems (Aversa et al., 2009; Annunziata et al., 2006, Apicella et al., 2010; Beaupre and Hayes, 1985; Reilly and Burstein, 1974; Reilly and Burnestain, 1975). The procedure is illustrated in the Figures from 2 to 5 . The external geometry of femur has been reconstructed by generating a three-dimensional volume that interpolates the CT scans (Fig. 2).

The results were then imported in the 3Matic software for surface and solid meshing optimization as indicated in Fig. 3.

Internal modeling of the entire femur has been realized by defining three-dimensional internal tethraedric meshing distribution and size optimization (Fig. 4).
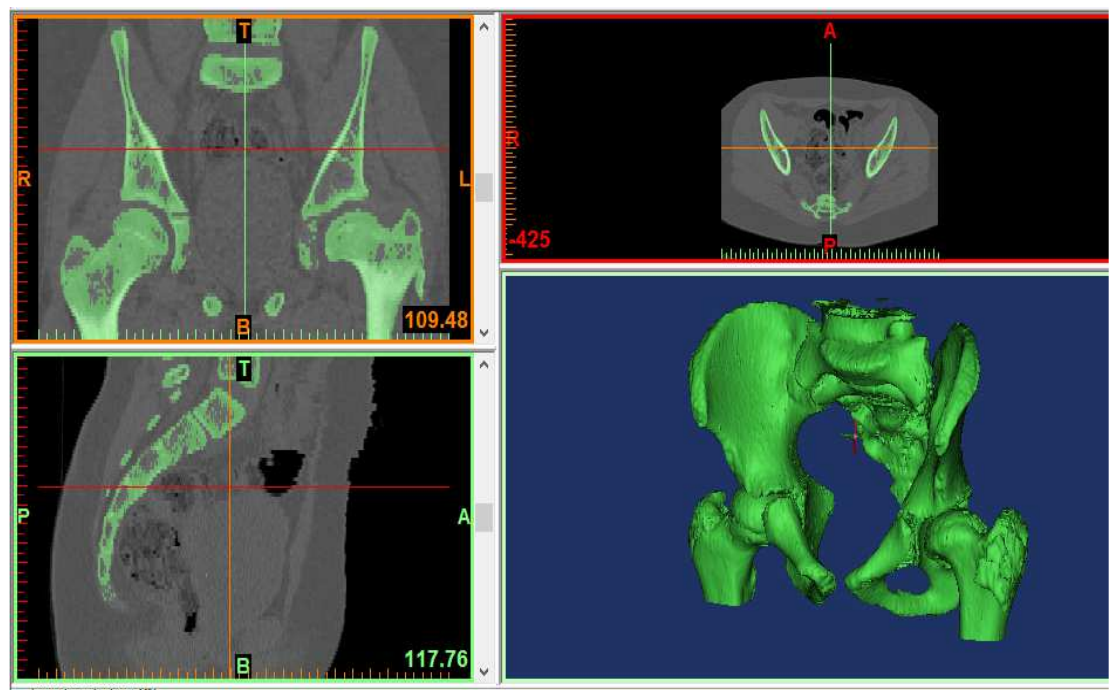

Fig. 1. Biofidel medical Image from Computerized Tomography (CT) of a patient pelvis: Point clouds raw data
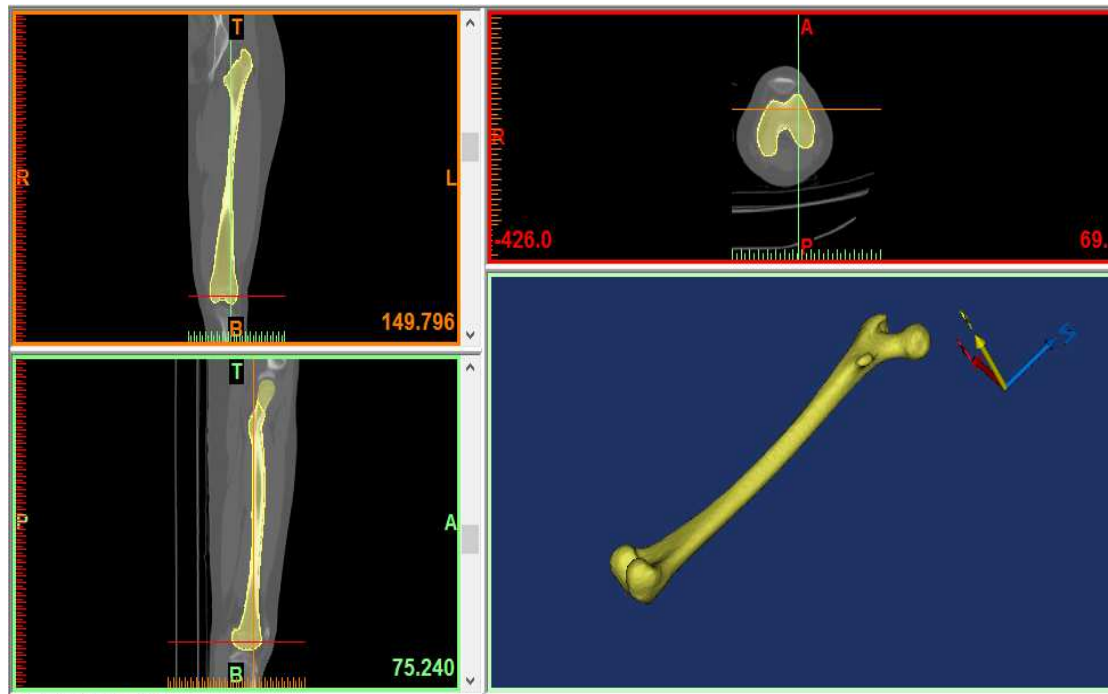

Fig. 2. CT segmentations and Biofidel 3D solid modelling: Transverse (upper right), medial (upper left), frontal (lower left) and bone solid reconstruction of a patient femur (lower right) 


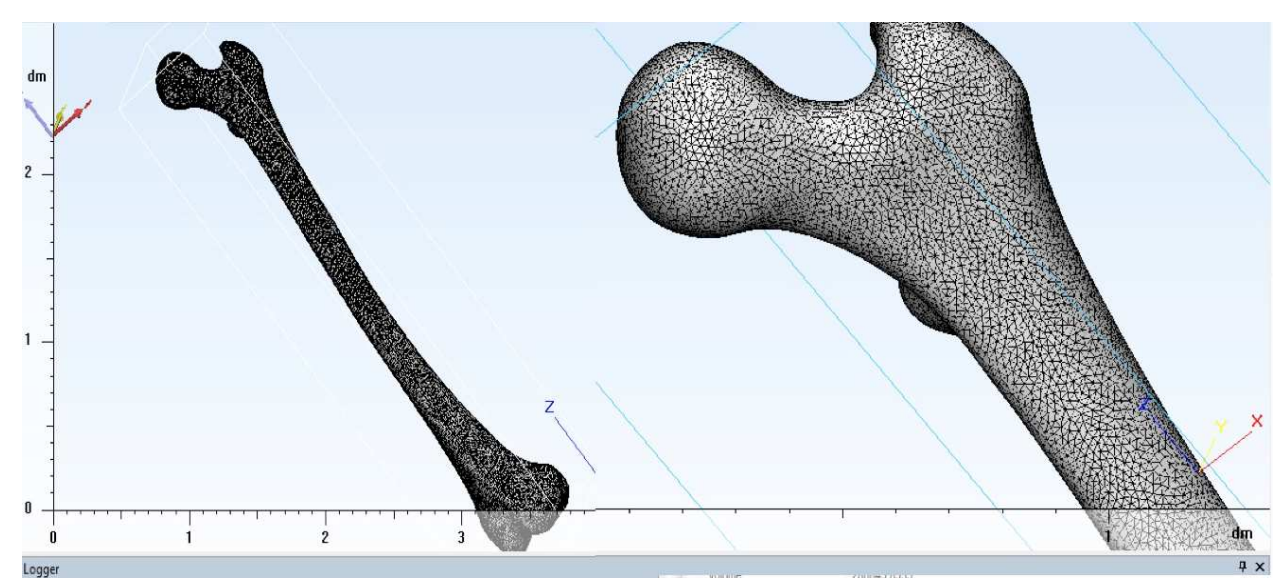

Fig. 3. Preliminary triangle surface meshing optimization of the biofidel patient femur model

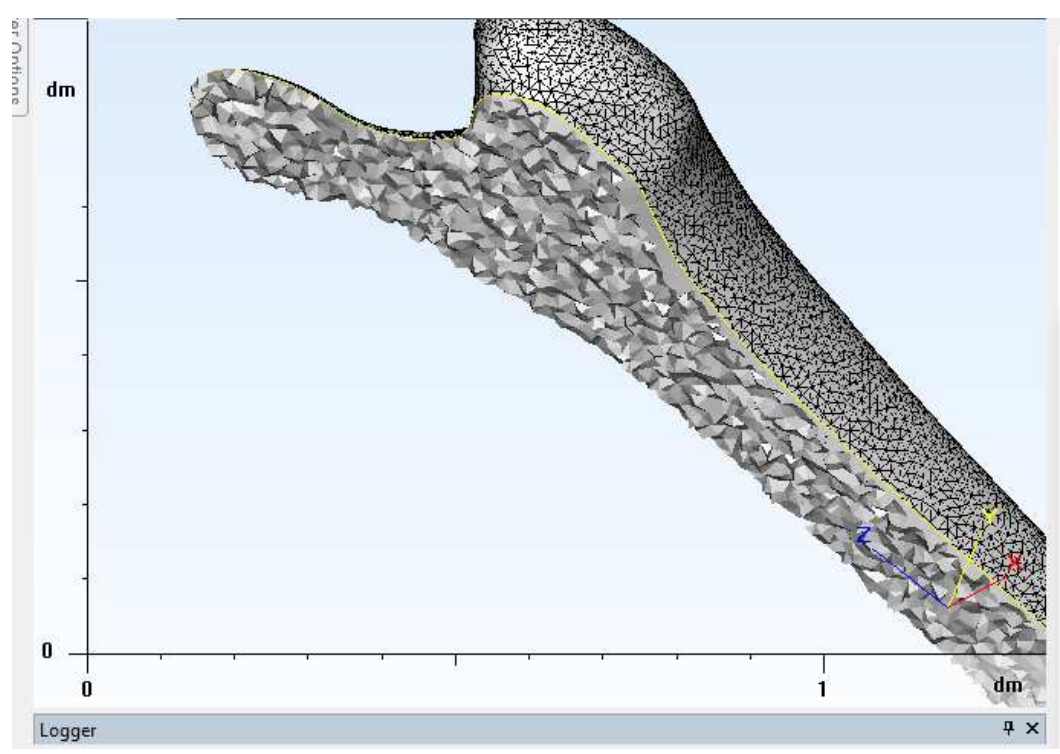

Fig. 4. Tethraedric 3D solid meshing optimization of the biofidel patient femur model (detail of the proximal end)
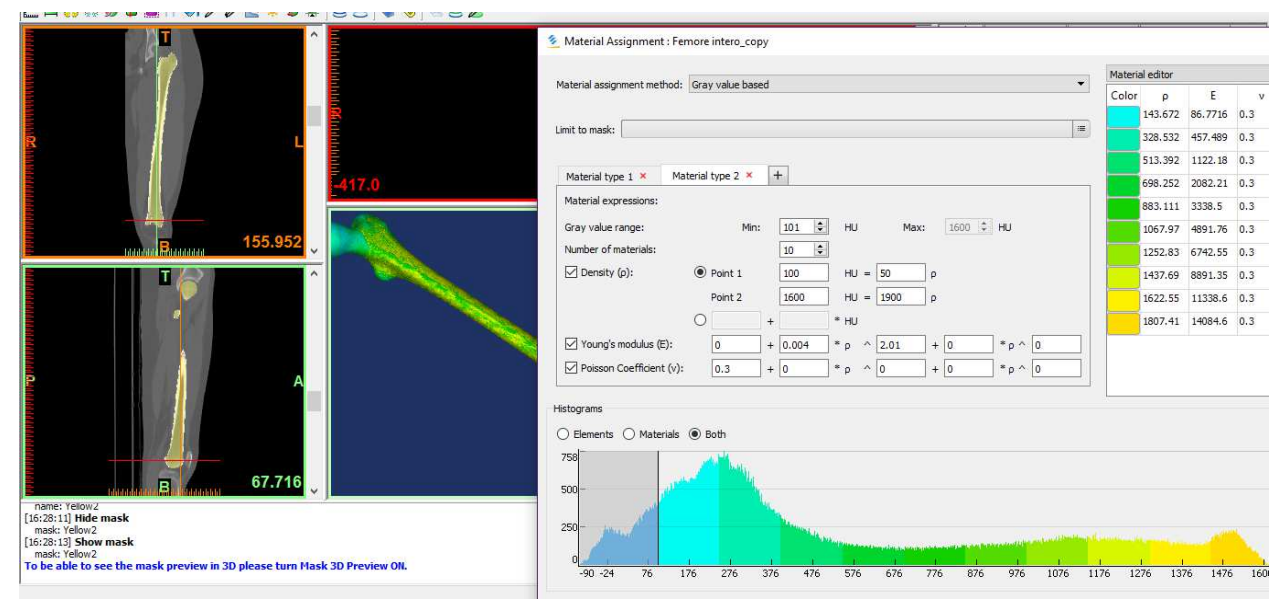

Fig. 5. Material properties definition associated to the patient femur cortical and trabecular bone densities (left) and material properties assignation to HU bone densities (right) 
The solid mesh elements have been successively associated to the bone densities measured according to the Hounsfield (HU) scale, which quantify the linear attenuation coefficients of X-rays in the tissues, and then assigned to the FEM model by the Mimics software (Fig. 5).

The evaluation of mechanical properties has been done considering the trabecular bone characteristics. The systems have been considered as transversally isotropic materials, with elastic and shear modulus expressed in terms of values of cortical bone and the values of elastic and shear modulus have been then evaluated multiplying those of cortical bone for the porosity defined for each tethraedric element by the Hounsfield (HU) densities scale. In this scale, the fat is worth about -110 , the muscle about 40 , the trabecular bone is in the range between 100 and 300 and the cortical bone extends beyond the trabecular bone values up to about 2000 .

By operating on the internal structure and bone trabecular morphology, which represents the oriented trabecular system of proximal end, the solid mesh elements representing the trabecular-oriented material properties have been assigned (Fig. 6) and the isotropic mechanical properties calculated as indicated in Table 1.

At each tethraedric mesh element has been assigned by the software a color corresponding, according to the color property map reported in Table 1 , to a specific mechanical isotropic mechanical property defined by the Hounsfield (HU) scale.

\section{Orthotropic Mechanical Properties Computation}

The morphology of the porosity characterizing trabecular bone structure is related to the kind of state stress acting on the system. The different typology of trabecular bone porosity has made evident by the femur head internal structure (Fig. 7).

The clear orientation of trabecular system observed in the lower left of Fig. 6, which is observed in the area of the epiphysis subjected to tensile stresses, implicates that the tensile stress state is oriented in that direction; the absence of any directionality (namely a spherical morphology) indicates the absence of trabecular orientation that occurs in the volumes where the stress state is compressive. From the mechanical properties stand point of view, it can be inferred that the oriented trabecular volumes are characterized by orthotropic material properties while non-oriented ones show an isotropic material behavior.

A mechanistic model of the hip proximal epiphysis has been proposed by Kummer (1986), which is related to the presence of isostatic lines characterizing the oriented trabecular systems, is reported in Fig. 8. These morphological differences are better appreciated by comparing trabecular bone of oriented and non-oriented regions (Fig. 7 and 8 ).

The previously CT computed values of bone densities have been then related to the isostatic lines of cortical and trabecular bone assigning to each tethraedric mesh element an orientation according to the stress isolines directions. This work defines a FEM model of human femur that could simulate geometrically the bone orthotropicity. The 3D solid and meshed FEM models have been exported in Solidworks software (Dassault Systèmes SolidWorks Corp.) to run the structural evaluations under specific biological loading conditions.
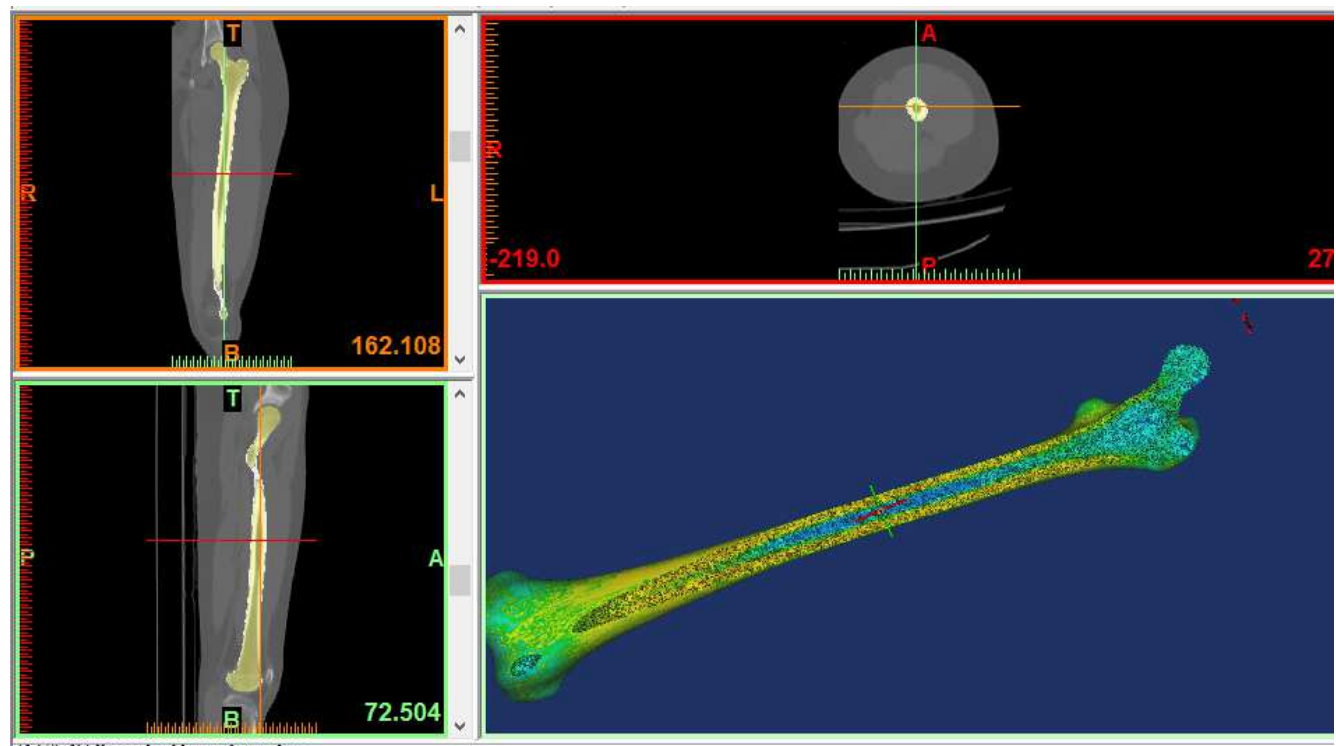

Fig. 6. Material properties definition according to Hounsfield scale associated to the patient femur cortical and trabecular bone densities: Medial (upper left), Transverse (upper right) and Frontal (lower right) sections and 3D solid sectioned femur FEM 


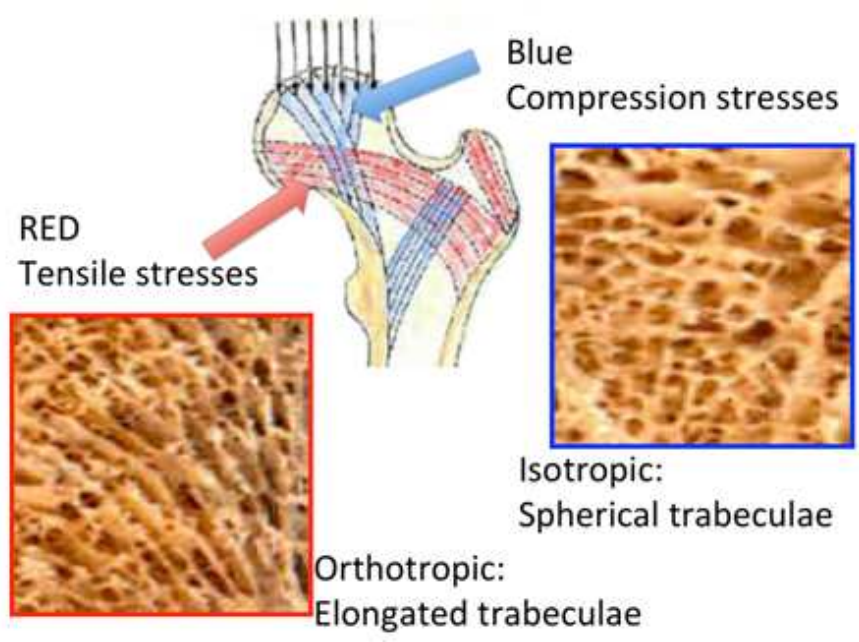

Fig. 7. Stress state and morphology in the femur proximal epiphysis cortical and trabecular bone

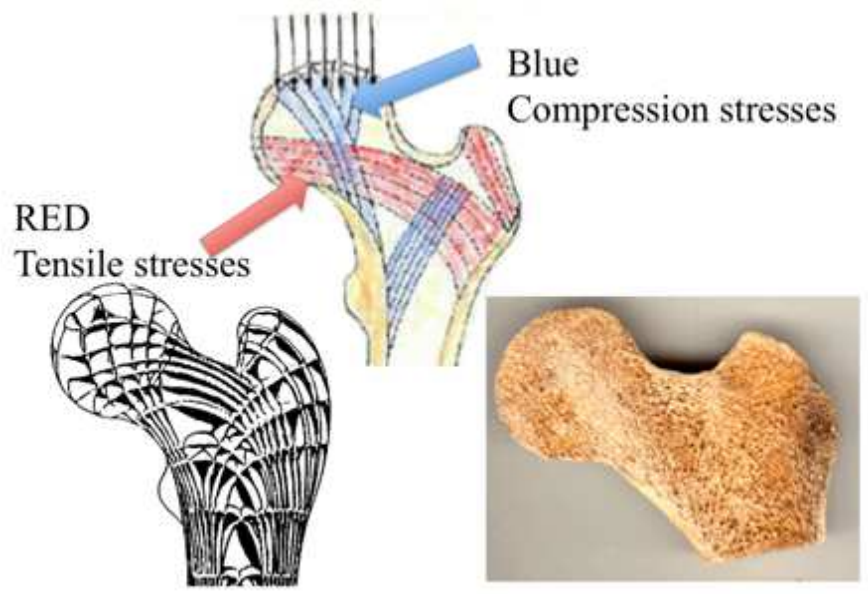

Fig. 8. Kummer iso-tension lines (1986) mechanistic model and trabecular bone densities and orientations in a sectioned femur head

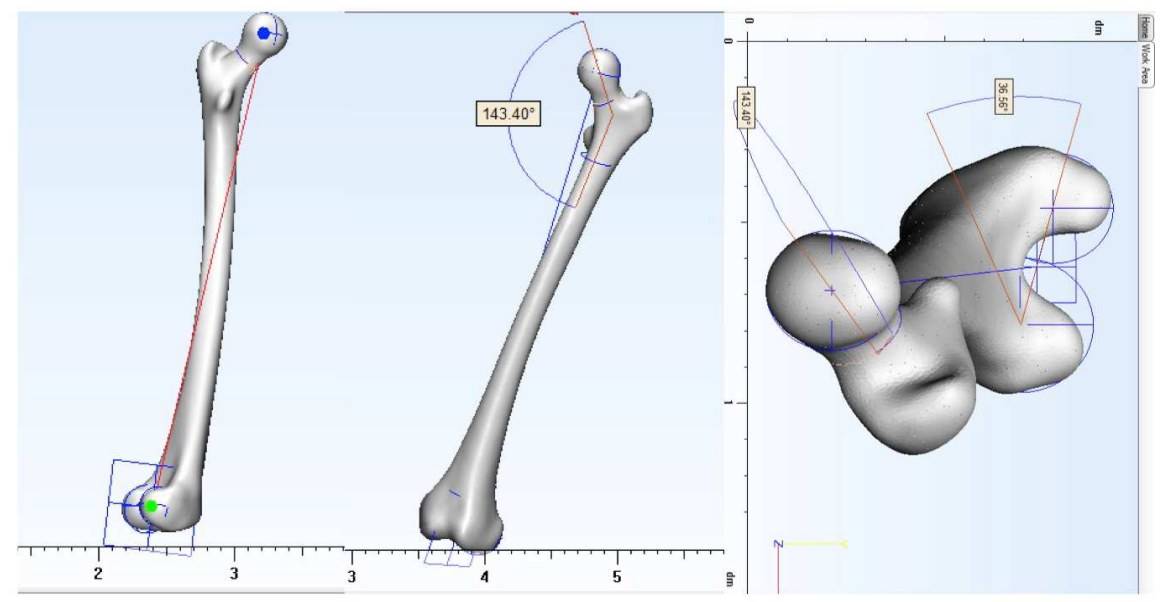

Fig. 9. Biometric analysis: Mechanical axis of the femur, Angle of the femoral neck $\left(143.40^{\circ}\right)$, Divergence of the neck axis with the axis of epicondyles $\left(36.65^{\circ}\right)$ 


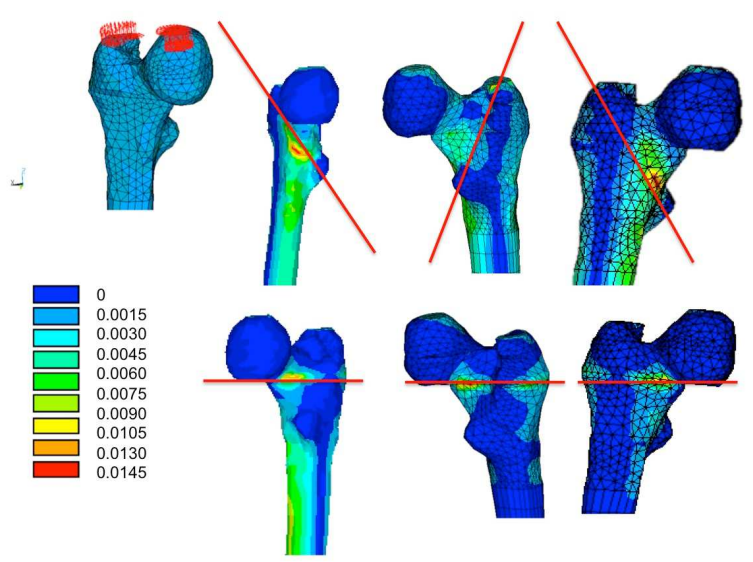

Fig. 10. Equivalent Von Mises strains in the proximal diaphysis for orthotropic (upper) and isotropic (lower) trabecular bone properties. Red lines: Potential pertrochanteric femur fracture planes
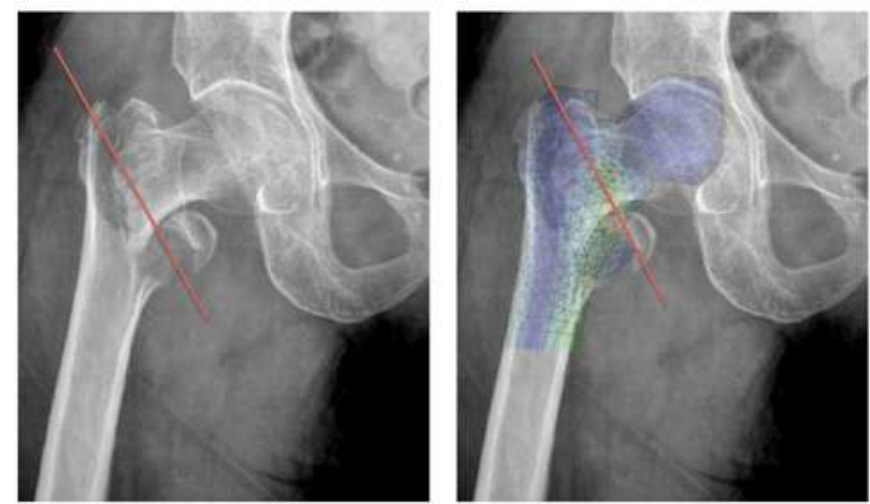

Fig. 11. CT and fracture plane (red line) of a pertrochanteric femur fracture (left) compared to the fracture plane and strain distribution evaluated from the biofidel Finite Element Analysis for orthotropic distribution of the trabecular bone (right)

Table 1. Isotropic mechanical properties assigned to the trabecular and cortical bone

\begin{tabular}{|c|c|c|c|}
\hline Material editor & \multicolumn{2}{l}{$v$} \\
\hline Color & $\rho$ & $E$ & \\
143.672 & 86.7716 & 0.3 \\
\hline 328.532 & 457.489 & 0.3 \\
\hline 513.392 & 1122.18 & 0.3 \\
\hline 698.252 & 2082.21 & 0.3 \\
\hline 883.111 & 3338.5 & 0.3 \\
\hline 1067.97 & 4891.76 & 0.3 \\
\hline 1252.83 & 6742.55 & 0.3 \\
\hline 1437.69 & 8891.35 & 0.3 \\
\hline & 1622.55 & 11338.6 & 0.3 \\
\hline & 1807.41 & 14084.6 & 0.3 \\
\hline & & & \\
\hline
\end{tabular}

\section{FEM Analysis: Isotropic and Orthotropic Models}

On the basis of 3D models two different material mechanical properties distribution have been developed and compared.

The first case considers the isotropic distribution of the mechanical properties over the entire femur while the second one assigns orthotropic mechanical properties to the proximal diaphysis (femur head).

\section{Biometric Analysis}

Before running the structural analysis it is necessary to define the personal characteristic biometric parameters of the patient femur-hip system. For this analysis was used the 3Matic software to:

- Identify the loading axis

- Identify the center of the femoral head ball (creating a sphere that mediates the surface selected)

- Define the center of the joint epicondyle and mechanical axis of rotation of the knee 
The following parameters.

Once the biometric parameters have been identified, before the structural analysis it is necessary to define physiological loads and constrains.

\section{Loads and Constrains}

The force equilibrium condition in the monopodalic posture has been considered as a limiting loading condition. This condition consists in the hip rotation equilibrium around the center of hip joint. It has been assumed that, in equilibrium, the sum of moment of body weight force and of gluteus muscular force is zero. It has been considered the patient body weight of 100 $\mathrm{Kg}$, a gluteus muscular force applied to the great trochanter of $1800 \mathrm{~N}$ and a joint reaction force of 2740 $\mathrm{N}$, which have been calculated from the equilibrium monopodalic equilibrium posture using the biometric parameters reported in Fig. 9. The equilibrium condition has been applied in the frontal plane, that is the plane defined by the mechanical axis and the hip joint center. Gluteus reaction force has been uniformly spread over 100 nodes of great trochanter region, while joint reaction force has been allocated on 50 nodes of the head (as indicated in the upper left of Fig. 10).

\section{Results and Discussion}

The study is aimed to define a procedure to biofidelly model the femur structural behavior. Von Mises strain criterion has been used to compare and validate the twotrabecular properties mechanical properties distributions. This energetic criterion can quantify the capability of the bone to withstand high loads (Fig. 10). The orthotropic model realizes a more uniform strains distribution and better mimics the action of the load on the diaphysis. The different structural behavior of the two models is also made evident by the Von Mises distribution of the strains, which are diverse at the back and frontal bone. Posteriorly, the strain distribution suggests the presence of flexure stress state, with maximum stress and strain distributed at the anatomical neck and great trochanter regions (upper part of Fig. 10). Furthermore, the orthotropic trabecular distribution is characterized by highest values of the strain distribution and energy at the femur of neck region; in this way, a more deformable region is detected in the anatomical region where the occurrence of pertrochanteric femur fractures are usually observed. This area, named the Ward Triangle, is the region where the trabecular bone density reaches its minimum and where the bone loss in aged people is higher. For this reason, the probability to start a femurneck fracture in this area is really high.

The Finite Element model adopting an orthotropic distribution of the trabecular mechanical properties is able to correctly predict the fracture behavior observed for these pertrochanteric femur fractures as can be inferred from the CT scan reported in the right part of Fig. 11. In the right part of Fig. 11, infact, the result of our analysis is overlying the actual CT image. The fracture plane observed for a real fracture coincides with the plane fracture evaluated from the biofidel model developed in this study.

\section{Conclusion}

A biofidel femur Finite Element Model has been developed from CT scans using specific combination of software's to correctly represent bone physiology and structural behavior. Proper identification of trabecular bone arrangement and distribution in the proximal diaphysis enabled modeling and definition of material properties. The faithful femur model proposed allows us to correctly account for non-isotropic properties to the proximal end explaining the critical structural role played by trabecular bone that should be taken into account in the design of new innovative prosthetic system.

The trabecular bone has been considered as a porous system, with a variable apparent density. By comparing the mechanical behaviour of the spongeous bone, which can be seen as a trabecular bone without pores, the trabecular system, which has been modeled as differently oriented parts, was characterized as an orthotropic materials while not oriented ones has been characterized as isotropic materials. A comparison between a FEM analysis on this model and on the model that considers the proximal end as an isotropic material shows that the orthotropic model simulates a more realistic stress distribution in the bone because permits to simulate the structural role played by the trabecular systems, detecting clearly a bone crisis region. A method to correctly approach the femur/neck fracture and the femur/prosthesis interface in a prosthesized bone have been than presented and it will be used in the design of new prosthetic systems.

\section{Acknowledgement}

Funds from Italian Ministry of University and Research PRIN 2008 and FIRB (Funds for Base Resarch) Futuro in ricerca 2008.

\section{Author's Contributions}

All the authors contributed equally to prepare, develop and carry out this manuscript.

\section{Ethics}

This article is original and contains unpublished material. The corresponding author confirms that all of the other authors have read and approved the manuscript and no ethical issues involved. 


\section{References}

Annunziata, M., R. Aversa, A. Apicella, A. Annunziata and D. Apicella et al., 2006. In vitro biological response to a light-cured composite when used for cementation of composite inlays. Dental Mater., 22: 1081-1085. PMID: 16387354

Apicella, D., R. Aversa, E. Ferro, D. Ianniello and A. Apicella, 2010. The importance of cortical bone orthotropicity, maximum stiffness direction and thickness on the reliability of mandible numerical models. J. Biomed. Mater. Res. Part B Applied Biomater., 93: 150-163. DOI: 10.1002/jbm.b.31569

Ashman, R.B., S.C. Cowin, W.C. Van Buskirk and J.C. Rice, 1984. A continuous wave technique for the measurement of the elastic properties of cortical bone. J. Biomechan., 17: 349-361. DOI: 10.1016/0021-9290(84)90029-0

Ashman, R.B. and J.Y. Rho, 1988. Elastic modulus of trabecular bone material. J. Biomechan., 21: 177-1781. DOI: 10.1016/0021-9290(88)90167-4

Aversa, R, D. Apicella, L. Perillo, R. Sorrentino and F. Zarone et al., 2009. Non-linear elastic threedimensional finite element analysis on the effect of endocrown material rigidity on alveolar bone remodeling process. Dental Mater., 25: 678-690. DOI: $10.1016 /$ j.dental.2008.10.015

Beaupre, G.S. and W.C. Hayes, 1985. Finite element analysis of a three-dimensional open-celled model for trabecular bone. J. Biomech. Eng., 107: 249-56. DOI: $10.1115 / 1.3138550$

Burnstein, A., D.T. Reilly and M. Martens, 1976. Aging of bone tissue: Mechanical properties. J. Bone Joint Surgery, 58: 82-86. PMID: 1249116

Carter, D.R. and W.C. Hayes, 1977. The compressive behavior of bone as a two-phase porous structure. J. Bone Joint Surgery, 59A: 954-962. PMID: 561786

Dalstyra M., R. Huiskes, A. Odgaard and L. Van Erning, 1993. Mechanical and textural properties of pelvic trabecular bone. J. Biomechan., 26: 349-361. DOI: 10.1016/0021-9290(93)90014-6

Gramanzini, M., S. Gargiulo, F. Zarone, R. Megna and A. Apicella et al., 2016. Combined microcomputed tomography, biomechanical and histomorphometric analysis of the peri-implant bone: A pilot study in minipig model. Dental Mater., 32: 794-806.

DOI: $10.1016 /$ j.dental.2016.03.025
Kummer, B., 1986. Biomechanical principles of the statistics of the hip joint. A critical appraisal of a new theory, Zeitschrift fur Orthopadie und Ihre Grenzgebiete, 124: 179-187.

DOI: $10.1055 / \mathrm{s}-2008-1044544$

Oh, I. and W.H. Harris, 1976. Proximal strain distribution in the loaded femur. An in vitro comparison of the distributions in the intact femur and after insertion of different hip-replacement femoral components. J. Bone Joint Surgery, 60: 75-85. PMID: 624762

Perillo, L., R. Sorrentino, D. Apicella, A. Quaranta and C. Gherlone et al., 2010. Nonlinear visco-elastic finite element analysis of porcelain veneers: A submodelling approach to strain and stress distributions in adhesive and resin cement. J. Adhesive Dent., 12: 403-413.

Reilly, D.T. and A.H. Burnestain, 1975. The elastic and ultimate properties of compact bone tissue. J. Biomechan., 8: 393-405. DOI: 10.1016/0021-9290(75)90075-5

Reilly, D. and A.H. Burstein, 1974. The mechanical properties of cortical bone. J. Bone Joint Surgery, 56: 1001-1021.

Rohlmann, A., U. Mossner, G. Bergmann and R. Kolbel, 1982. Finite-element-analysis and experimental investigation of stresses in a femur. J. Biomed. Eng., 4: 241-246. DOI: 10.1016/01415425(82)90009-7

Sorrentino, R., R. Aversa, V. Ferro, T. Auriemma and F. Zarone et al., 2007. Three-dimensional finite element analysis of strain and stress distributions in endodontically treated maxillary central incisors restored with diferent post, core and crown materials. Dent Mater., 23: 983-993.

DOI: $10.1016 /$ j.dental.2006.08.006

Sorrentino, R., D. Apicella, C. Riccio, E.D. Gherlone and F. Zarone et al., 2009. Nonlinear visco-elastic finite element analysis of different porcelain veneers configuration. J. Biomed. Mater. Res. Part B Applied Biomater., 91: 727-736. DOI: 10.1002/jbm.b.31449

Gottesman, T. and Z. Hashin, 1980. Analysis of viscoelastic behaviour of bones on the basis of microstructure. J. Biomechan., 13: 89-96. DOI: 10.1016/0021-9290(80)90182-7 